\title{
Rapid and accurate identification of species belonging to the Candida parapsilosis complex by real-time PCR and melting curve analysis
}

\author{
Constantin Hays, Chantal Duhamel, Vincent Cattoir and Julie Bonhomme \\ Microbiology Laboratory, Côte de Nacre University Hospital, Caen, France
}

Correspondence

Julie Bonhomme

bonhomme-j@chu-caen.fr

Received 28 September 2010 Accepted 22 December 2010
Candida parapsilosis is the second most frequent Candida species isolated from blood cultures. Since 2005, C. parapsilosis has been divided into three distinct species based on genetic traits: Candida parapsilosis, Candida metapsilosis and Candida orthopsilosis. The aim of this study was to develop a rapid real-time PCR assay able to distinguish these closely related species via a melting curve analysis. This identification method was optimized by using reference strains and well-characterized clinical isolates of Candida species. A single set of consensus primers was designed to amplify a 184 bp portion of the $S A D H$ gene in order to identify species based on the unique melt profile resulting from DNA sequence variations from each species of the complex. PCR products were detected with SYBR Green fluorescent dye and identification was established by melting curve analysis. For validation of the technique, a total of 116 clinical isolates, phenotypically identified as $C$. parapsilosis, were tested by real-time PCR and results were further compared with PCR-RFLP patterns of the SADH gene, used as the reference method. The melting curve analysis of amplified DNA could differentiate between C. parapsilosis $\left(83.5^{\circ} \mathrm{C}\right)$, C. metapsilosis $\left(82.9^{\circ} \mathrm{C}\right)$ and C. orthopsilosis $\left(82.1^{\circ} \mathrm{C}\right)$, with a sensitivity and specificity comparable to those of the reference method. One hundred and fourteen C. parapsilosis and two C. orthopsilosis isolates were identified among the clinical isolates. This method provides a simple, rapid and reliable identification of species belonging to the C. parapsilosis complex. This novel approach could be helpful for clinical and epidemiological investigations.

\section{INTRODUCTION}

Among Candida species, Candida parapsilosis is the second most frequently isolated from bloodstream infections in many regions of the world, especially in Latin America and Asia, while it is also common in Europe (Almirante et al., 2006). C. parapsilosis emerged in the 1990s and its incidence is still increasing (Playford et al., 2010). This is partly due to the massive usage of medical devices, notably among immunocompromised patients, and to the selective pressure exerted by the intensive use of azoles and more recently of echinocandins (Bassetti et al., 2009; Forrest et al., 2008; Trofa et al., 2008). C. parapsilosis is also known to be a nosocomial yeast responsible for bloodstream infections, mainly in intensive care units, transmitted by the hands of healthcare workers (van Asbeck et al., 2007). Moreover, its ability to form biofilms on medical implants, like Candida albicans, confers increased resistance to antifungal agents, leading to a real therapeutic problem (Kuhn et al., 2002; Růžička et al., 2007).

Since 2005, C. parapsilosis has been considered to be a species complex, including three distinct species based on genetic criteria: C. parapsilosis, Candida metapsilosis and
Candida orthopsilosis (Tavanti et al., 2005). Epidemiological and clinical features corresponding to each species have been poorly documented since their phenotypic identification remains unreliable. Therefore, the rapid identification of Candida species in clinical laboratories appears essential for the management of patients with candidaemia.

The aim of this study was to develop a rapid real-time PCR assay able to distinguish these three closely related species via a melting curve analysis. After optimization of this technique, we tested 116 clinical isolates, previously identified by conventional methods as C. parapsilosis, and compared with PCR-RFLP patterns of the SADH gene, used as the reference method.

\section{METHODS}

Clinical isolates and reference strains. A total of 116 clinical isolates identified as C. parapsilosis using the Vitek 2 YST card or API ID $32 \mathrm{C}$ (bioMérieux) were collected from 79 patients hospitalized in the Caen University Hospital from October 2006 to September 2010. They were recovered from blood (36), central venous catheter (22), urine (15), respiratory tract (10), faeces (13) or others (20). C. parapsilosis 
ATCC 22019, C. metapsilosis CBS 10907 and C. orthopsilosis CBS 10906 were included in the study as reference strains. Three clinical strains, identified as $C$. orthopsilosis by sequencing, were also studied (gift from M.-E. Bougnoux, Hôpital Necker-Enfants Malades, Paris, France). Other Candida species of clinical interest were used to ensure the technique specificity: C. albicans, Candida glabrata, Candida tropicalis, Candida krusei, Candida kefyr, Candida lusitaniae, Candida dubliniensis, Candida famata, Candida colliculosa and Candida sphaerica. A clinical isolate identified as Saccharomyces cerevisiae and the Lodderomyces elongiporus reference strain CBS 2605 were also tested. Each strain was cultured in YPD liquid medium ( $2 \%$ glucose, $2 \%$ peptone, $1 \%$ yeast extract) overnight at $30{ }^{\circ} \mathrm{C}$ with agitation and stored at $-80{ }^{\circ} \mathrm{C}$.

DNA extraction. Yeast genomic DNA was extracted with the MasterPure Yeast DNA Purification kit (Epicentre Biotechnologies) according to the manufacturer's instructions.

Real-time PCR and melting curve analysis. After aligning the $S A D H$ sequences of the three species, a single set of consensus primers was carefully designed to amplify a $184 \mathrm{bp}$ portion of the gene, in order to identify species based on the unique melt profile resulting from DNA sequence variations from each $C$. parapsilosis complex species. The $25 \mu \mathrm{l}$ reaction mixture consisted of $12.5 \mu \mathrm{l}$ SYBR Green PCR Master Mix $2 \times$ (Qiagen), $2.5 \mu \mathrm{l}$ forward and reverse primers $\left(10 \mu \mathrm{mol} 1^{-1}\right)$ (SADH-F $5^{\prime}$-GCTGCGGCTTCAACTGATGC-3' and SADH-R 5'-CTTGGTCACGAGCCTCC-3'), $5.5 \mu$ distilled water and $2.0 \mu \mathrm{l}$ DNA sample (approx. $150 \mathrm{ng}$ ). Real-time PCR and melting curve analysis were carried out using a Rotor-Gene Q thermocycler (Qiagen). The experimental conditions used were as follows: $5 \mathrm{~min}$ at $95{ }^{\circ} \mathrm{C}$, followed by 40 cycles of $10 \mathrm{~s}$ at $95{ }^{\circ} \mathrm{C}, 30 \mathrm{~s}$ at $64{ }^{\circ} \mathrm{C}$ and $20 \mathrm{~s}$ at $72{ }^{\circ} \mathrm{C}$. Melting curves were generated after the last extension step; the temperature was ramped from $55{ }^{\circ} \mathrm{C}$ to $95{ }^{\circ} \mathrm{C}, 0.5{ }^{\circ} \mathrm{C}$ every $5 \mathrm{~s}$. Melting curves were analysed by the Rotor-Gene 1.7 software (Qiagen).

PCR-RFLP analysis. Results for each strain identified by real-time PCR and melting curve analysis were compared to those obtained with PCR-RFLP used as the reference method, as previously described (Asadzadeh et al., 2009). Briefly, primers SADHF2 (5'-TGGATTGTGGGGAYAATTATGT- $3^{\prime}$ ) and SADHR2 (5'-GTCAATACRGCATCAGTTGAAGC-3') were used to amplify a $310 \mathrm{bp}$ fragment of the $S A D H$ gene. Subsequently, PCR products were digested with the restriction enzyme BanI.

Statistical analysis. Student's $t$ test was used to establish differences between test groups. A $P$-value of $<0.05$ was considered significant.

\section{RESULTS AND DISCUSSION}

The primers SADH-F and SADH-R were designed to amplify a portion of the $S A D H$ gene, with a DNA $\mathrm{G}+\mathrm{C}$ content difference between the three species (C. parapsilosis, 45.1 mol\%; C. metapsilosis, $43.5 \mathrm{~mol} \%$; and C. orthopsilosis, 41.3 mol\%). This difference allowed species identification with three significantly different melting temperatures: $83.5 \pm$ $0.19{ }^{\circ} \mathrm{C}, 82.9 \pm 0.15{ }^{\circ} \mathrm{C}$ and $82.1 \pm 0.25{ }^{\circ} \mathrm{C}$ for C. parapsilosis, C. metapsilosis and C. orthopsilosis, respectively $(P<0.05)$ (Fig. 1). A difference of $0.7^{\circ} \mathrm{C}$ maximum in the melting peak temperatures could be observed between different runs, but not within the same run. This disparity probably resulted from variations in the temperature profile created by the thermocycler, requiring the use of reference strains DNA in each set as positive controls. No amplification was observed with C. albicans, C. glabrata, C. tropicalis, C. krusei, C. kefyr, C. lusitaniae, C. dubliniensis, C. famata, C. colliculosa, C. sphaerica, S. cerevisiae or L. elongiporus (specificity, $100 \%$ ). The latter species is very close phenotypically and genetically to C. parapsilosis, formerly considered as its teleomorph, resulting in misidentification by conventional methods (Lockhart et al., 2008a). Concerning the PCR-RFLP reference method, the BanI-digested PCR products generated two fragments of 166 and $144 \mathrm{bp}$, two fragments of 268 and $42 \mathrm{bp}$, and one undigested fragment of $310 \mathrm{bp}$ for $C$. parapsilosis, C. metapsilosis and C. orthopsilosis, respectively, as expected.

Since C. parapsilosis was considered a species complex and divided into three species, several identification methods have been developed, based on PCR-RFLP (Tavanti et al., 2005; Asadzadeh et al., 2009) and internal transcribed spacer sequencing (Gomez-Lopez et al., 2008; Borman et al., 2009). An amplification fragment length polymorphism method has also been used not only to confirm species identification, but also to evaluate genetic relatedness of C. parapsilosis (Tavanti et al., 2010), C. metapsilosis (Hensgens et al., 2009) and C. orthopsilosis (Tavanti et al., 2007). All these techniques are time-consuming and not adapted for routine diagnosis. In 2009, a real-time PCR with melting temperature

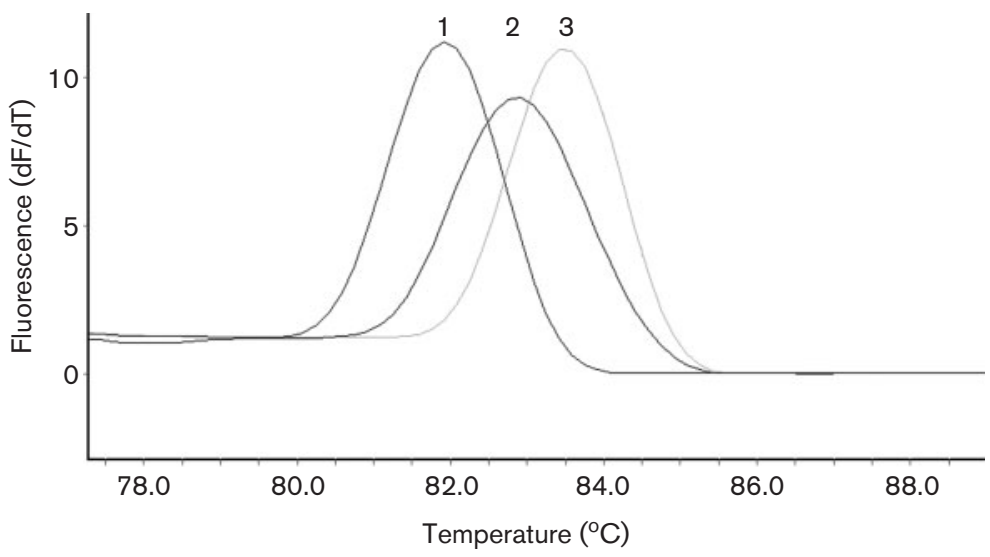

Fig. 1. Characteristic melting peaks with genus-specific forward and reverse primers for identification of C. parapsilosis (peak numbered 3), C. metapsilosis (peak numbered 2) and C. orthopsilosis (peak numbered 1). 
analysis was developed to identify six Candida species of clinical interest, including C. parapsilosis complex species (Khan et al., 2009). However, this technique has not yet been optimized to enable species-level identification of the $C$. parapsilosis complex. In our study, the melting curve analysis of amplified DNA could differentiate between the three species of the $C$. parapsilosis complex with a sensitivity and specificity comparable to those of the PCR-RFLP reference method.

The 116 clinical isolates, previously identified by conventional methods as $C$. parapsilosis, were correctly amplified and detected with SYBR Green fluorescent dye, as were the three C. orthopsilosis clinical strains. Based on the melting curve analysis, $114(98.3 \%)$ and two $(1.7 \%)$ were identified as C. parapsilosis and C. orthopsilosis, respectively, with $100 \%$ correlation with the PCR-RFLP reference method. The three C. orthopsilosis clinical strains were correctly identified with the two methods. No C. metapsilosis was identified among these clinical isolates. Although epidemiological data are still rare, this species distribution seemed unusual. Indeed, among C. parapsilosis complex species, the percentage of $C$. orthopsilosis and C. metapsilosis was low compared to that in Asia (11\% of C. orthopsilosis and $2.6 \%$ of C. metapsilosis), Europe and the Middle East (3.5\% of C. orthopsilosis and $2.9 \%$ of C. metapsilosis), South America $(10.9 \%$ of C. orthopsilosis and $1.3 \%$ of $C$. metapsilosis) and North America (5\% of C. orthopsilosis and $0.6 \%$ of C. metapsilosis) (Lockhart et al., 2008b). Europe is generally considered to be the region with the higher rate of C. metapsilosis (Lockhart et al., 2008b).

In this study, $56 \%$ of the $C$. parapsilosis isolates were from patients over 60 years of age, with a median age of 55 years. $C$. parapsilosis is usually described as an important pathogen in children and neonates. We found that $8.9 \%$ of the $C$. parapsilosis isolates were from children under 1 year, consistent with the majority of publications (Filioti et al., 2007).

This method provides a simple, rapid (less than $3 \mathrm{~h}$, including DNA extraction) and reliable identification of species belonging to the $C$. parapsilosis complex. In addition, it seems to be cost-effective since no specific probes are needed in this assay. This novel approach may be helpful for clinical and epidemiological investigations. To our knowledge, we have developed the first rapid and accurate real-time PCR for species differentiation within the C. parapsilosis complex.

\section{ACKNOWLEDGEMENTS}

We are sincerely grateful to Marie-Elisabeth Bougnoux for her pertinent advice and for providing us with reference strains and $C$. orthopsilosis clinical strains.

\section{REFERENCES}

Almirante, B., Rodríguez, D., Cuenca-Estrella, M., Almela, M., Sanchez, F., Ayats, J., Alonso-Tarres, C., Rodriguez-Tudela, J. L.,
Pahissa, A. \& the Barcelona Candidemia Project Study Group (2006). Epidemiology, risk factors, and prognosis of Candida parapsilosis bloodstream infections: case-control population-based surveillance study of patients in Barcelona, Spain, from 2002 to 2003. J Clin Microbiol 44, 1681-1685.

Asadzadeh, M., Ahmad, S., Al-Sweih, N. \& Khan, Z. U. (2009). Rapid molecular differentiation and genotypic heterogeneity among Candida parapsilosis and Candida orthopsilosis strains isolated from clinical specimens in Kuwait. J Med Microbiol 58, 745-752.

Bassetti, M., Ansaldi, F., Nicolini, L., Malfatto, E., Molinari, M. P., Mussap, M., Rebesco, B., Bobbio Pallavicini, F., Icardi, G. \& Viscoli, C. (2009). Incidence of candidaemia and relationship with fluconazole use in an intensive care unit. J Antimicrob Chemother 64, 625-629.

Borman, A. M., Linton, C. J., Oliver, D., Palmer, M. D., Szekely, A., Odds, F. C. \& Johnson, E. M. (2009). Pyrosequencing analysis of 20 nucleotides of internal transcribed spacer 2 discriminates Candida parapsilosis, Candida metapsilosis, and Candida orthopsilosis. J Clin Microbiol 47, 2307-2310.

Filioti, J., Spiroglou, K., Panteliadis, C. P. \& Roilides, E. (2007). Invasive candidiasis in pediatric intensive care patients: epidemiology, risk factors, management, and outcome. Intensive Care Med 33, 12721283.

Forrest, G. N., Weekes, E. \& Johnson, J. K. (2008). Increasing incidence of Candida parapsilosis candidemia with caspofungin usage. J Infect 56, 126-129.

Gomez-Lopez, A., Alastruey-Izquierdo, A., Rodriguez, D., Almirante, B., Pahissa, A., Rodriguez-Tudela, J. L., Cuenca-Estrella, M. \& the Barcelona Candidemia Project Study Group (2008). Prevalence and susceptibility profile of Candida metapsilosis and Candida orthopsilosis: results from population-based surveillance of candidemia in Spain. Antimicrob Agents Chemother 52, 1506-1509.

Hensgens, L. A., Tavanti, A., Mogavero, S., Ghelardi, E. \& Senesi, S. (2009). AFLP genotyping of Candida metapsilosis clinical isolates: evidence for recombination. Fungal Genet Biol 46, 750-758.

Khan, Z., Mustafa, A. S. \& Alam, F. F. (2009). Real-time LightCycler polymerase chain reaction and melting temperature analysis for identification of clinically important Candida spp. J Microbiol Immunol Infect 42, 290-295.

Kuhn, D. M., Chandra, J., Mukherjee, P. K. \& Ghannoum, M. A. (2002). Comparison of biofilms formed by Candida albicans and Candida parapsilosis on bioprosthetic surfaces. Infect Immun 70, 878-888.

Lockhart, S. R., Messer, S. A., Pfaller, M. A. \& Diekema, D. J. (2008a). Lodderomyces elongisporus masquerading as Candida parapsilosis as a cause of bloodstream infections. J Clin Microbiol 46, 374-376.

Lockhart, S. R., Messer, S. A., Pfaller, M. A. \& Diekema, D. J. (2008b). Geographic distribution and antifungal susceptibility of the newly described species Candida orthopsilosis and Candida metapsilosis in comparison to the closely related species Candida parapsilosis. J Clin Microbiol 46, 2659-2664.

Playford, E. G., Nimmo, G. R., Tilse, M. \& Sorrell, T. C. (2010). Increasing incidence of candidaemia: long-term epidemiological trends, Queensland, Australia, 1999-2008. J Hosp Infect 76, 46-51.

Růžička, F., Holá, V., Votava, M. \& Tejkalová, R. (2007). Importance of biofilm in Candida parapsilosis and evaluation of its susceptibility to antifungal agents by colorimetric method. Folia Microbiol (Praha) 52, 209-214.

Tavanti, A., Davidson, A. D., Gow, N. A., Maiden, M. C. \& Odds, F. C. (2005). Candida orthopsilosis and Candida metapsilosis spp. nov. to replace Candida parapsilosis groups II and III. J Clin Microbiol 43, 284-292.

Tavanti, A., Hensgens, L. A., Ghelardi, E., Campa, M. \& Senesi, S. (2007). Genotyping of Candida orthopsilosis clinical isolates by 
amplification fragment length polymorphism reveals genetic diversity among independent isolates and strain maintenance within patients. J Clin Microbiol 45, 1455-1462.

Tavanti, A., Hensgens, L. A., Mogavero, S., Majoros, L., Senesi, S. \& Campa, M. (2010). Genotypic and phenotypic properties of Candida parapsilosis sensu strictu strains isolated from different geographic regions and body sites. BMC Microbiol 10, 203.
Trofa, D., Gácser, A. \& Nosanchuk, J. D. (2008). Candida parapsilosis, an emerging fungal pathogen. Clin Microbiol Rev 21, 606-625.

van Asbeck, E. C., Huang, Y.-C., Markham, A. N., Clemons, K. V. \& Stevens, D. A. (2007). Candida parapsilosis fungemia in neonates: genotyping results suggest healthcare workers hands as source, and review of published studies. Mycopathologia 164, 287293. 